Décadrages Décadrages

cinéma, à travers champs Cinéma, à travers champs

37-38 | 2018

Lionel Rogosin

\title{
Dans la rue, sous les draps : le New York de Lionel Rogosin
}

\section{Paula J. Massood}

Traducteur : Sylvain Portmann

\section{OpenEdition \\ Journals}

\section{Édition électronique}

URL : https://journals.openedition.org/decadrages/1245

DOI : $10.4000 /$ decadrages. 1245

ISSN : 2297-5977

\section{Éditeur}

Association Décadrages

\section{Édition imprimée}

Date de publication : 1 mars 2018

Pagination : 12-27

ISBN : 978-2-9700963-4-4

ISSN : 2235-7823

\section{Référence électronique}

Paula J. Massood, «Dans la rue, sous les draps : le New York de Lionel Rogosin », Décadrages [En ligne], 37-38 | 2018, mis en ligne le 02 février 2021, consulté le 04 avril 2022. URL : http:// journals.openedition.org/decadrages/1245; DOI : https://doi.org/10.4000/decadrages.1245 
Paula J. Massood

(traduction de l'américain par Sylvain Portmann)

\section{Dans la rue, sous les draps: le New York de Lionel Rogosin}

1 Jonas Mekas, «Notes sur le nouveau cinéma américain", dans Jonas Mekas, Le Cinéma de la nouvelle génération, trad. Christian Lebrat, Coll. Les Cahiers de Paris expérimental, Paris, Paris expérimental, 2002, p. 22.

2 John Pruitt, «Jonas Mekas: A European Critic in America», dans David E. James, To Free the Cinema: Jonas Mekas and the New York Underground, Princeton, Princeton University Press, I992, p. 53 [notre trad.].

3 Jonas Mekas, «Notes sur le nouveau cinéma américain», op. cit., pp. 22-23. Concernant les films de Levitt et Meyers, voir Paula J. Massood, Making a Promised Land: Twentieth-Century African American Photography and Film, New Brunswick, Rutgers University Press, 20I2, pp. 94-IIO.
DANS le nUméro DE PRINTEMPS i962 de la revue Film Culture, Jonas Mekas définit les conditions d'un nouveau type de cinéma. Dans un article intitulé «Notes sur le nouveau cinéma américain», l'auteur proclame qu'il n'est ni en train d'essayer «de construire des théories abstraites», ni de «juger l'œuvre du nouvel artiste à partir des outils de la Culture», mais qu'il compte «examiner largement et de première main les idées, styles et réalisations de ce nouveau cinéma» ${ }^{1}$. L'article de Mekas, qui pose les bases de sa vision d'une nouvelle génération de réalisateurs new-yorkais indépendants, propose à la fois un aperçu historique du cinéma indépendant américain depuis la fin des années 1940, un inventaire des réalisateurs actifs et un repérage de procédés stylistiques et thématiques. L'une des principales composantes de ce Nouveau cinéma américain est ce que John Pruitt désigne comme un réalisme «glissant», un «critère décisif $»^{2}$ pour Mekas à ce moment-là.

Influencé d'une part par le néoréalisme italien et par le réalisme bazinien d'autre part, Mekas situe les racines du Nouveau cinéma américain dans des films new-yorkais réalistes et sociaux, ceux de Helen Levitt, Janice Loeb, James Agee et Sidney Meyers; plus particulièrement In The Street (Helen Levitt, E.-U., I948), un court métrage mettant en scène des enfants qui jouent à Harlem, et The Quiet One (Sidney Meyers, E.-U., I948), une docufiction de long métrage portant sur un jeune AfroAméricain de Harlem envoyé dans une école de redressement pour jeunes délinquants. Pour Mekas, ces films «sont aux prises avec un sujet réaliste, les deux emploient des non-acteurs; les deux ont été tournés sur le lieu même. [...] Et ils sont tous les deux spontanés dans l'action et dans la caméra ce qui les distingue de leurs prédécesseurs documentaristes [...] et des films expérimentaux réalisés à la même époque ${ }^{3}$. Pour l'auteur, ils illustrent parfaitement la proposition d'Agee selon laquelle les 
films doivent ne pas être des «documentaires, mais des ouvres de pure fiction, joués contre, au sein et en collaboration avec une réalité inattendue et non inventée » ${ }^{4}$. Les films, et plus particulièrement The Quiet One, atteignent aussi un niveau d'engagement politique jamais égalé par les productions antérieures comprenant des personnages afro-américains et qui critiquent, de façon parfois suggérée, les institutions politiques et sociales qui ont abouti à l'abandon de populations intra-muros, en particulier la jeunesse noire.

Cette insistance sur l'engagement social pousse Mekas à mettre les films de Lionel Rogosin en avant - Rogosin avait alors réalisé On the Bowery (1956) et Come Back, Africa (1960) - pour leur forme qui, associant un réalisme à une «conscience sociale», intègrent, tout comme The Quiet One auparavant, des aspects documentaires à la fiction. Selon Mekas, «l'une des contributions de Rogosin au nouveau cinéma était une effective dramatisation de la réalité, l'utilisation de scènes de la vie réelle dans un canevas organisé, planifié. Cette union du documentaire et du dramatique lui a permis d’enregistrer la vérité de la situation des lèvres mêmes des gens qui en réalité vivaient eux-mêmes ces situations ${ }^{5}{ }^{5}$. Il n'y a aucun doute que les films de Rogosin, plus particulièrement $O n$ The Bowery, conviennent parfaitement à une certaine forme de réalisation new-yorkaise du moment, et il est indubitable que le réalisateur luimême est une figure centrale du Nouveau cinéma américain à ses débuts, ce sur quoi je reviendrai plus avant. Ce qui est moins clair, c'est la manière dont ses films plus tardifs, tel Black Fantasy (E.-U., I972), renforcent cette position. La suite de cet article sera consacrée à la façon dont On the Bowery et Black Fantasy représentent deux moments distincts, bien qu'interconnectés, de l'histoire du Nouveau cinéma américain; le premier étant associé à une forme de réalisme social tandis que le second tend vers une approche de la réalité plus expérimentale dans sa forme.

\section{Dans la rue: le Nouveau cinéma américain et New York}

Ecrivant à la même époque que Mekas (1962), Harris Dienstfrey tente également d'identifier les films et les réalisateurs associés aux productions indépendantes basées à New York. Dans un article de la revue Commentary intitulé «Le Nouveau cinéma américain», l'auteur dessine les contours de ce qui caractérise selon lui un "phénomène culturel spontané»: des documentaires à petit budget, des courts métrages, qui
4 Jonas Mekas, «Notes sur le nouveau cinéma américain»,op. cit, p. 23.

5 Id., p. 24 . 
6 Harris Dienstfrey, «The New American Cinema», Commentary Magazine, $\mathrm{I}^{\mathrm{er}}$ juin i962 (en ligne: www.commentary magazine.com/articles/the-new-american -cinema/) [notre trad.]. Dernière consultation le 20 juin 2017 .

7 Ibid. Quelques années plus tard, le personnage principal de The Cool World (Shirley Clarke, E.-U., I963) se rend également à Coney Island afin de prouver à sa compagne que New York se situe sur un océan. Tout comme Joey dans Little Fugitive, Duke, le protagoniste du film de Clarke, parcourt les plages, les attractions et les arcades de l'endroit. Pour Joey comme pour Duke, Coney Island fournit une expérience bien différente de celles qu'offrent respectivement leurs quartiers de Brooklyn et Harlem.

8 J.Mekas, op. cit., p. 24.

9 Lionel Rogosin, A Rebel with a Cause: The Autobiography of Lionel Rogosin, Maverick Independent Filmmaker, sans date, archives privées de Lionel Rogosin, Angers, France. se présentent avec «des techniques hypernaturalistes et des sujets qui traitent des troubles populaires de l'Amérique contemporaine ${ }^{6}$. A la différence de Mekas, qui fait remonter le mouvement aux ouvres de Levitt, Loeb et Meyers, Dienstfrey soutient que Little Fugitive (Morris Engel, E.-U., I953) constitue le premier exemple de ce qui peut être considéré comme du Nouveau cinéma américain. Disputes quant aux origines mises à part, tous les films - In the Streets, The Quiet One, et Little Fugitive - ont des points communs, notamment leur recours à des acteurs non professionnels, un tournage in situ (plus particulièrement lorsqu'il s'agit de filmer des extérieurs), et le mélange de procédés traditionnellement réservés soit à la fiction, soit à la non-fiction. Tout comme In the Streets et The Quiet One, Little Fugitive se concentre sur le quotidien d'enfants à New York, présentant ainsi la ville et ses quartiers (respectivement Harlem et Brooklyn), à travers les yeux de ses plus jeunes habitants. Le résultat, surtout avec le film d'Engel, c'est que «des histoires dénuées de dramaturgie deviennent partie intégrante de l'actualité qui les entoure ${ }^{7}$, dans ce cas le voyage d'un jeune homme à Coney Island. A la différence de The Quiet One, qui explorait les effets du racisme et de la pauvreté sur une famille afro-américaine de Harlem, le film d'Engelévite de gloser sur «les problèmes sociaux de l'Amérique contemporaine» et adopte au contraire un regard plus proche des expériences d'un enfant livré à lui-même dans la ville après que son frère aîné lui a joué un tour qui se retourne contre lui. Il ne faudra pas attendre longtemps avant que d'autres réalisateurs associés au Nouveau cinéma américain ne fixent leur attention sur des sujets sociaux: pauvreté, racisme ou autres fléaux sociaux tels que la toxicomanie. C'est là où Rogosin, qui selon Mekas «a apporté au cinéma indépendant une forte note de conscience sociale» ${ }^{8}$, rentre en ligne de compte.

Dans ses mémoires inédites, Rogosin se réclame des néoréalistes italiens et de Robert Flaherty, plus précisément de Man of Aran (L'Homme d'Aran, R.-U., I934), comme source d'inspiration pour On the Bowery, une étude des bas quartiers de New York présentée à travers les histoires personnelles de trois de leurs résidents, Ray, Gorma ( Doc») et Frank ${ }^{9}$. Le film mêle aux images documentaires des habitants du quartier et de ses recoins des scènes écrites et relativement dirigées, basées sur la vie des personnages, afin d'accéder aux histoires et aux expériences de ces trois hommes, leur servant ainsi d'intermédiaire tout en leur conférant une 


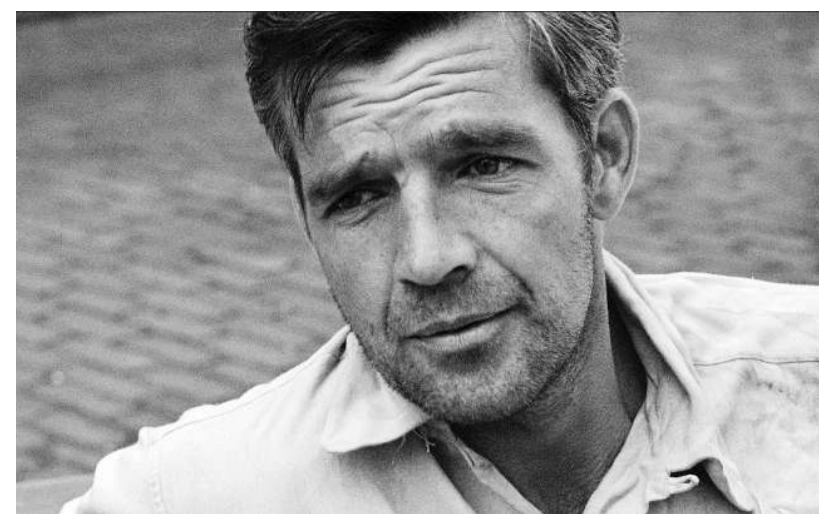

part d'humanité. De nombreuses scènes sont constituées de longs plans et emploient une caméra fixe pour documenter l'intérieur des bars et des pensions du Bowery, qui sont alors la destination finale pour nombre de clochards de la ville, les accros et les indigents. Les plans d'intérieur sont montés avec des scènes de rue montrant les hommes et les femmes des bas-fonds, buvant, déambulant, cherchant du travail, dormant devant des maisons ou sur des pas-de-porte, ou, comme dans une scène saisissante, buvant l'alcool frelaté d'une boîte d'alcool à brûler Sterno. Les sujets sont souvent cadrés en gros plan, l’image de leurs visages abîmés étant plus éloquente qu'aucun dialogue relatant l'histoire crue de leurs $\operatorname{vies}(\text { fig. I })^{10}$. La résultante de cette combinaison de scènes de rue et d'intérieur, de scènes de foules et de regards intimes sur des individus, vise à instiller une ambiance de désespoir et d'empathie; les personnages du film sont certes ravagés par l'alcool et les maladies mentales, mais leurs voix les humanisent. Plutôt qu'ostracisés comme de misérables saoulons, Ray, Doc et Frank deviennent des personnes à part entière.

Tout comme Flaherty, qui demandait souvent aux personnes qu'il filmait de rejouer des gestes anciens afin de les présenter comme une représentation typique de leurs vies, Rogosin et son équipe, qui comprenait l'écrivain Mark Sufrin et le chef opérateur Richard Bagley, ont travaillé avec leurs trois personnages afin d'obtenir un rendu naturaliste au travers de leurs histoires respectives. Beaucoup de scènes ont été improvisées (autre caractéristique du Nouveau cinéma américain), partant
1/ On the Bowery (1956)

10 De nombreux gros plans ressemblent aux photographies de Walker Evans, Berenice Abbott et Helen Levitt, qui tous trois visaient à capturer des aspects variés de la vie et de la persévérance américaines durant la première moitié du $\mathrm{Xx}^{\mathrm{e}}$ siècle. 
11 H. Dienstfrey, op.cit., n.p. [notre trad.].

12 Lionel Rogosin, «Interpréter la réalité (Notes sur l'esthétique et les pratiques du jeu improvisé)» [r96o], trad. Faye Corthésy, dans ce numéro, p. I46.

13 Dans New York in the 5os (New York, St. Martin’s Griffin, I999), Dan Wakefield relate une soirée où Baldwin et Bagley boivent des verres ensemble dans la White Horse Tavern, accompagnés de deux femmes blanches. D'autres clients, «enragés à la vue d'une femme blanche assise à côté de [Baldwin]», s'en prennent aux deux hommes. Wakefield poursuit en suggérant que c'est à ce moment que Baldwin «a su qu'il ne serait jamais en sécurité dans l’Amérique blanche» (id., p. I40 [notre trad.]). de sujets basés sur le quotidien de ces hommes. A titre d'exemple, une scène nous montre Ray, ancien cheminot, raconter à Doc sa vie passée sur les routes; on apprend qu'il a fait un court passage dans l'armée avant de mener une vie itinérante à travailler sur les rails et à chercher des emplois d'un jour afin de nouer les deux bouts. Un tel traitement du récit, selon Dienstfrey, «ne jouit d'aucune marque au sol renseignant l'acteur sur la position qu'il doit occuper. Les films [du Nouveau cinéma américain] utilisent la caméra afin d'enregistrer une action de la façon la plus naturelle possible. Les techniques [...] sont employées au service de la dure réalité, et pas le contraire " ${ }^{11}$. Rogosin décrit son travail en avançant que lorsqu’il s’agit de «restituer la réalité de façon spontanée et lui donner vie, l'implication ne se limite [...] pas à simplement choisir des personnes d'un certain milieu. Celles-ci doivent pouvoir être elles-mêmes et s'exprimer de leur propre manière, tout en étant en accord avec les abstractions et les thèmes que vous, en tant que réalisateur, pouvez voir en eux. [...] Pour explorer la vie intérieure de personnes dans un milieu particulier, je crois [...] que la méthode que j’ai suivie dans mes films est plus profonde et vraie» ${ }^{12}$.

Que l'approche esthétique et thématique d'On The Bowery ressemble à celle de The Quiet One ne doit pas surprendre puisque ces films sont tous deux le résultat d'un milieu particulier au sein duquel les idées et les personnes se rencontrent et se fréquentent à New York. C’est un fait relativement connu que Rogosin n'est pas un réalisateur chevronné lorsqu'il tourne son premier film. En effet, il déclare qu’On the Bowery lui sert de terrain d'entraînement avant de réaliser Come Back, Africa, le documentaire qu'il compte faire depuis qu'il avait été témoin d'atrocités pendant son service dans la marine lors de la Seconde Guerre mondiale. Selon Rogosin, il a commencé à apprivoiser l'usage de la caméra Bolex i6mm alors qu'il cherchait à se concentrer sur un sujet adéquat et qu'il le trouve sur le Bowery. Le destin a voulu qu'il rencontre alors le chef opérateur Richard Bagley et l'écrivain Mark Sufrin à la White Horse Tavern, dans le West Village, un lieu réputé où se rencontrent certains écrivains, notamment James Baldwin ${ }^{13}$, Jane Jacobs et Jack Kerouac.

Avant sa collaboration avec Rogosin, Bagley a tourné The Quiet One de Meyers et, selon certaines sources, aurait travaillé auparavant dans l'industrie du cinéma en Italie. Il a ainsi apporté à la production d'On the Bowery des techniques cinématographiques et narratives simi- 
laires: tournage in situ, acteurs non professionnels, avec un intérêt prononcé pour la réalité quotidienne. Sufrin est un écrivain free-lance qui a quelque expérience dans les films de non-fiction. Il aide Rogosin à tracer les grandes lignes des personnages en fonction de leurs vécus et apparaît au rang des producteurs du film. Le montage est commencé par Helen Levitt - photographe, réalisatrice d'In the Streets et signant en partie l'image de The Quiet One (également amie de Bagley) - et achevé par Carl Lerner, monteur de films à petit budget tournés à New York, notamment The Big Break (Joseph Strick, E.-U., I953) ${ }^{14}$. En résumé, le film de Rogosin bénéficient de bon nombre de talents qui sont sur la même longueur d'onde et au bon moment (le milieu des années 1950) au bon endroit (le centre-ville de New York).

Bien qu'issu d'une famille d'industriels aisée, Rogosin, autodidacte et sortant de Yale, est loin d'un dilettante dénué de convictions éthiques. Il se consacre entièrement à son sujet et à l'esthétique du cinéma indépendant à petit budget. Ce constat se manifeste de façon exemplaire dans l'effort qu'il met à réaliser son second long métrage, Come Back, Africa, un film anti-apartheid tourné sur place à Johannesburg et à Sophiatown, en Afrique du Sud, qui offre une distribution mixte, mettant en scène Noirs et Blancs, et qui emprunte plusieurs directions esthétiques (en particulier le mélange d'images documentaires et de fiction) à l'instar de son premier film. Le film a été tourné clandestinement à Sophiatown - puisqu'il est interdit de filmer dans les quartiers ségrégués -, Rogosin prétendant qu'il produit là un documentaire de voyage à caractère musical. Le film est acclamé à l'étranger, et plus spécialement en Europe (où il est projeté au Festival du film de Venise), tandis qu'il est à peine remarqué aux Etats-Unis.

Rogosin est coutumier des difficultés à trouver des distributeurs ou des exploitants pour ses films qui ne se conforment pas aux conventions narratives hollywoodiennes. On the Bowery remporte par exemple le Grand Prix du documentaire et du court métrage au Festival de Venise en 1956 , et peu après un Academy Award britannique. Le film ne sort aux Etats-Unis qu'à partir du début de l’année 1957 et durant une courte période d'exploitation au $55^{\text {th }}$ Street Playhouse à New York, après quoi il est nominé pour un Academy Award. Malgré son succès, le film échoue à être distribué largement. De la même manière, Come Back, Africa, après avoir reçu de nombreuses récompenses dans des festivals, a des
14 Par la suite, Lerner montera I2 Angry Men (Douze hommes en colère, Sidney Lumet, E.-U., I957) et Klute (Alan J. Pakula, E.-U., I97I). Il réalisera également l'adaptation à l'écran de Black Like Me (I964) de John Howard Griffin. 
15 Collectif, «The First Statement of the New American Group», dans P. Adams Sitney (éd.), Film Culture Reader, New York, Cooper Square Press, 2000, p. 80 [notre trad.]. La première publication de cette déclaration apparaît dans Film Culture, n²2-23, été I96I, Pp. I3I-I33.

16 Id., p. 8 o. difficultés à être distribué aux Etats-Unis. Cette situation encourage Rogosin à acquérir ce qui allait devenir le Bleecker Street Cinema en I960. Le cinéma, qui projette des films indépendants, des films d’art et des reprises, est le lieu de la première de Come Back, Africa, au printemps de la même année. Il fonctionne aussi, au moins pour une courte période au début des années I960, comme un lieu de diffusion des films associés à Mekas ou à d'autres réalisateurs new-yorkais.

Le Groupe du Nouveau cinéma américain (New American Cinema Group) ne s'arrête pas à la liste des réalisateurs et des styles dressée par Mekas dans ses «Notes sur le nouveau cinéma américain». Il s'agit d'un groupe d'une vingtaine de réalisateurs engagés dans un cinéma non industriel (à nouveau: contre Hollywood) constitué par Mekas et par le producteur Lewis Allen à la fin de l'année i96o. Ce rassemblement comprend Rogosin, Peter Bogdanovich, Emile de Antonio, Shirley Clarke, Gregory Markopoulos, Argus Speare Juilliard et d'autres qui pensent qu'après des années d'«une manifestation inconsciente et sporadique», le «temps [est] venu de se rassembler» ${ }^{15}$ pour une juste cause. Dans le numéro de printemps i96i de Film Culture, le groupe formule sa première déclaration, un billet d'opinion déclarant leur mépris pour un «cinéma officiel» qui est «moralement corrompu, esthétiquement révolu, thématiquement superficiel, inévitablement lassant» ${ }^{16}$. A sa place, les membres proposent un cinéma d'«expression personnelle», libre de censure, détaché du poids des gros budgets et libéré des contraintes des systèmes en vigueur de distribution et d'exploitation. Afin de permettre l'exploitation de cinémas indépendants, le groupe propose un centre de distribution coopératif (dirigé par Emile de Antonio et relié à des salles telles que le Bleecker Street Cinema) et la création d'un festival de film de la côte Est dédié au Nouveau cinéma. Film Culture, tout comme d'autres publications telles que The Village Voice (pour laquelle Mekas est critique de cinéma), leur servent à annoncer publiquement des projections ainsi que d'autres activités du groupe.

La carrière de Rogosin en tant que réalisateur se raréfie durant les années I960, puisqu'il ne réalise qu'un long métrage et deux courts, mais il maintient pourtant son engagement envers les membres du groupe et d'autres réalisateurs indépendants, tout d'abord à travers sa gestion du Bleecker Street Cinema. Bien que la coopérative de distribution initialement proposée par le Groupe du Nouveau cinéma américain ne se concré- 


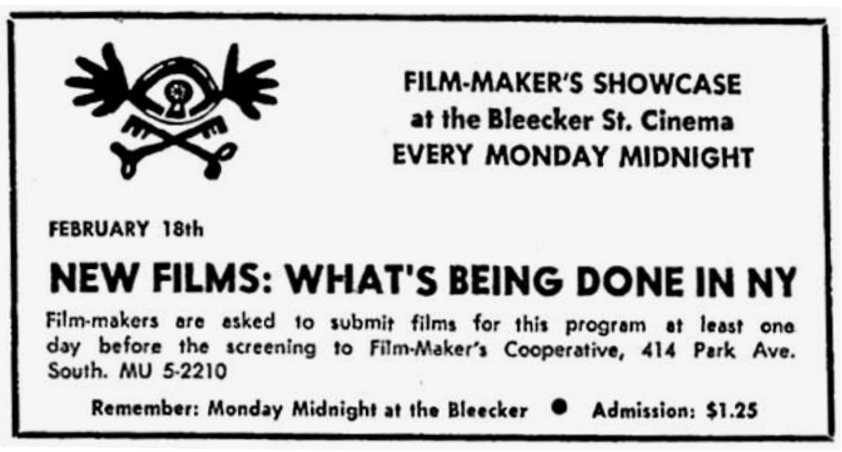

tise jamais véritablement, Mekas, accompagné par Shirley Clarke, Stan Brakhage (qui rejoint le groupe peu après) et par d'autres, fondent en I962 la Filmmaker's Cooperative, un organe de distribution «non exclusif, non discriminatoire ${ }^{17}$, géré par les réalisateurs eux-mêmes. Cette coopérative commence à offrir dès le début des années i960 une sélection d'œuvres indépendantes à certains cinémas de Manhattan, dont le Bleecker Street Cinema, qui présente des séances de minuit, à partir de films des membres de la coopérative, et des programmes ouverts afin de permettre aux réalisateurs locaux de montrer leur travail (fig.2). A cette période, Rogosin termine également un autre film, Good Times, Wonderful Times (G.-B., 1965) et fonde Impact Films en 1966, sa propre maison de distribution. Impact Films est initialement créée afin de distribuer Good Times, Wonderful Times, un film de compilation contre la guerre qui contient des images rares en provenance du monde entier. La compagnie distribue également des films indépendants venant d'autres pays à l'intention des universités et autres organisations culturelles sur le sol étasunien, un public prêt à accueillir Good Times, Wonderful Times, qui lui peine à trouver un distributeur, tout comme les films précédents du réalisateur. Comme nous l'avons relevé, mis à part quelques courts métrages, Rogosin ne retourne pas à la réalisation avant le début des années I970 lorsqu'il tourne Black Roots (E.-U., I970) et Black Fantasy. Ces films marquent aussi le retour du réalisateur à un intérêt pour les relations interraciales. Cette fois, pourtant, il tourne ses caméras vers New York et non pas vers l'étranger.
2/ Publicité pour une séance au Bleecker Street Cinema dans le Village Voice (14 février 1963, p. 25)

17 D. E. James, op. cit., p. Io [notre trad.]. 
18 L'ethnomusicologue Alan Lomax et sa fille, Anna Lomax, apparaissent au générique en tant que consultants musicaux pour le film. Il est fort probable que Lomax père ait contribué au rassemblement des musiciens pour le projet.

\section{Sous les draps : un retour à New York}

Durant le début des années i970, Rogosin tourne une trilogie explorant les différents aspects de la vie afro-américaine: Black Roots, Black Fantasy, et Woodcutters of the Deep South (E.-U., I973). Parmi ces trois films, les deux premiers se passent à New York et offrent un regard sur les effets des relations interraciales de l'Amérique urbaine tels qu'ils sont ressentis de plein fouet par ses habitants (enregistrant une fois de plus «la vérité de la situation des lèvres mêmes des gens qui en réalité vivaient eux-mêmes dans ces conditions»). Black Roots, par exemple, est un court métrage mettant en scène un groupe de musiciens de blues - comprenant Jim Collier, Révérend Gary Davis, Larry Johnson, Flo Kennedy - assis dans une pièce et se racontant des histoires de leur passé (reliées plus spécifiquement à la manière dont le racisme anti-Noir les a personnellement affectés), tout en jouant de la musique ${ }^{18}$. Avec le mélange de plans d'intérieur relativement statiques à des scènes de rue plus dynamiques, le film poursuit la technique que Rogosin emploie dès le début d’On the Bowery. Pour les intérieurs, par exemple, les musiciens sont filmés assis autour d'une table grâce à trois relativement longs plans stables. Il y a peu de gros plans, même lorsque l'attention se concentre sur une histoire individuelle. Souvent, lorsqu'un musicien joue ou chante, une coupe est faite sur un montage d'images d'extérieur, dans des rues où apparaît

Black Roots (1973)
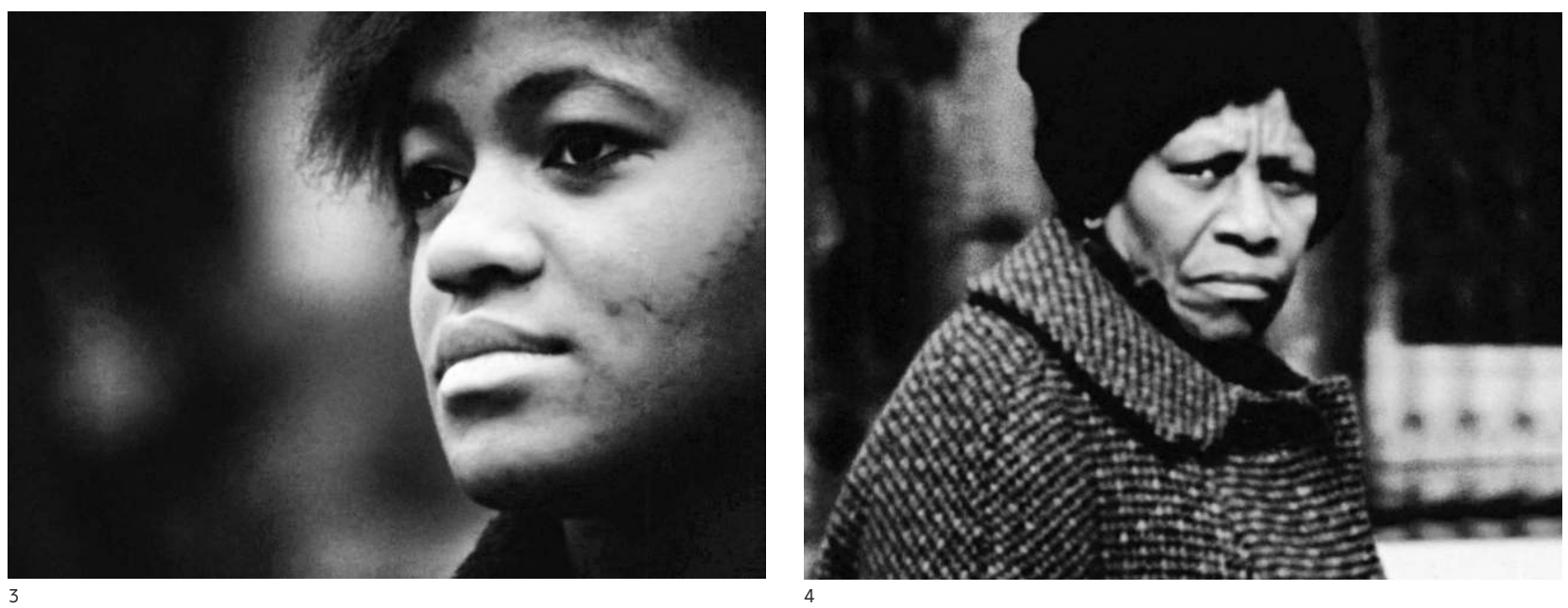
toute une variété de Noirs new-yorkais, dont bon nombre sont filmés en gros plan (fig. 3 et 4). Le procédé consiste à relier les cibles individuelles du racisme, dont il est question avec les musiciens, à la collectivité noire, et par là d'humaniser les personnes concernées ${ }^{19}$.

Tandis que Black Roots explore globalement le racisme anti-Noir, Black Fantasy fait figure d'étude plus intimiste et politisée de relations sexuelles interraciales. En ce sens, le film s'aligne sur les intérêts d'autres réalisateurs plus ou moins associés au Groupe du Nouveau cinéma américain, incluant John Cassavetes (Shadows, E.-U., I958; 1959) et Shirley Clarke (The Cool World, et Portrait of Jason, E.-U.,I967) qui font souvent référence, de façon implicite ou explicite, à des couples interraciaux ou plus généralement aux relations interraciales ${ }^{20}$. Pour Black Fantasy, Rogosin travaille à nouveau avec Jim Collier, l'un des musiciens qui apparaissent dans Black Roots, afin de restituer une dimension psychologique dans le racisme anti-Noir qui faisait défaut selon lui dans le film tourné auparavant. Tout comme le film précédent, Black Fantasy alterne des histoires personnelles avec des images documentaires prises dans les rues de New York. De plus, rompant avec le précédent style de Rogosin, le film comprend des moments expérimentaux de «fantaisie poétique ${ }^{21}$ où l'image et le son convergent afin de générer ce qui pourrait être décrit comme un rêve fiévreux de frustration sexuelle, de désir et de danger. En effet, le film est davantage une exploration expérimentale du désir sexuel manufacturé et réel qu'un documentaire linéaire sur l'histoire du racisme aux Etats-Unis. Ce changement de style est très vraisemblablement une résultante du sujet même, à quoi il faut ajouter le contact de Rogosin avec l'avant-garde cinématographique américaine, via ses relations issues de ce qui reste du Groupe du Nouveau cinéma américain (principalement des réalisateurs expérimentaux) et les films montrés au Bleecker Street Cinema.

Lorsque le Groupe du Nouveau cinéma américain énonce sa déclaration d'intentions en I96I, ses membres sont des documentaristes, des réalisateurs de fiction indépendants, des réalisateurs expérimentaux, ainsi que des producteurs, des distributeurs et des exploitants. Durant la décennie qui suit, un nombre important de membres d'abord associés au groupe, et plus particulièrement ceux engagés dans la réalisation de fiction, se distancient des postulats initiaux. Citons, à titre d'exemple, Rogosin, qui certes persévère dans ses documentaires sociaux, mais
19 Un procédé similaire de montage d'images de rue est visible dans The Cool World de Shirley Clarke, où l'histoire d'un jeune membre de gang à Harlem est ponctuée d'explorations visuelles et musicales de gens et de places du quartier. Pour une analyse plus poussée, voir P. J. Massood, Making a Promised Land, op. cit., plus spécialement pp. II4-I25.

20 Il est également envisageable de comparer l'esthétique de Black Roots avec The Connection (Shirley Clarke, E.-U., I96r), du moins dans l'attention portée sur un groupe de musiciens rassemblés en un lieu. Le film, basé sur une pièce de théâtre homonyme de Jack Gelber et interprété par le Living Theatre, est censuré dans l'Etat de New York pour son usage d'un langage vulgaire. Après plusieurs procès, le film parvient finalement à être montré à New York.

21 Entrée Black Fantasy du catalogue d'Impact Films (I973, p. 27). 
Pruitt, op. cit., p. 57 [notre trad.].

23 D. E. James, op.cit., p. Io [notre trad.]. Dans son introduction à To Free the Cinema, James dépeint la position de Mekas face à l'avant-garde américaine, allant d'un rejet initial à son adoption; l'auteur signale également l'arrestation de Mekas pour obscénité après avoir projeté Flaming Creatures (E.-U., I963) de Smith en ig64. de manière moins intensive, ainsi que Cassavetes (qui ne fait pas partie du groupe initial mais dont le film Shadows est cité dans la déclaration) et Bogdanovich qui déménagent à Hollywood, ou encore Clarke, qui fait quelques films qui tournent dans le circuit des films d'art avant de revenir à l'expérimental en réalisant des courts métrages vidéo et des performances artistiques. Les membres restants et ceux qui, comme Robert Breer, Jack Smith, et Stan Brakhage, commencent à travailler étroitement avec Mekas dans les années i960, sont avant tout des artistes d'avant-garde. Un tel revirement, selon Pruitt, est reconnu par Mekas lui-même qui est «forcé de reconnaître que le film réaliste indépendant ne se développe pas - et que si cette forme doit continuer, elle ne le fera malheureusement qu'en se compromettant entre l'art et l'industrie» ${ }^{22}$. David James remarque également qu'en 1963 «l'underground atteint son plein essor et jouit d'une visibilité sociale - voire de notoriété - sans précédent grâce à des œuvres dans lesquelles la tradition du réalisme social associé à New York se substitue bientôt à des extravagances sexuelles bizarres: les films de Ron Rice, Ken Jacobs et surtout Jack Smith, vite rejoints par les premières réalisations d'Andy Warhol - ce que Mekas nommait dans les colonnes du Village Voice du < cinéma baudelairien \» ${ }^{23}$. Comme mentionné plus haut, bien que Rogosin soit moins impliqué dans la poursuite des pratiques plus expérimentales du Groupe du Nouveau cinéma américain, il est en contact avec leurs travaux et, comme Black Fantasy le suggère, en subit l’influence, plus précisément par son caractère «baudelairien».

L'examen de deux séquences indique la manière dont Black Fantasy diffère des œuvres antérieures de Rogosin. En témoignent déjà les dix premières minutes du film, qui donnent le ton de l'œuvre à venir. Le film s'ouvre sur des images accompagnées de tambours sur la bande-son, montrant un Afro-Américain pourchassé par deux policiers blancs à travers les rues et les terrains vagues de la ville. Rapidement, les policiers l'attrapent et commencent à le frapper. C'est à ce moment que le point de vue passe de la troisième personne à celle de l'homme en train de se faire battre. La musique d'accompagnement cède alors la place à une chanson dont la voix masculine fait écho aux images, dont voici les paroles: «Viens me chercher, bourreau. Lance ta corde sur mon chemin. Lance ta corde sur mon chemin. [...] Viens me chercher, bourreau. J'ai pas peur de mourir. [...] Tu vas m’assassiner pour qui je suis». 
Une fois le générique terminé, l'image revient sur le même homme feignant de courir au ralenti dans une rue et au son extradiégétique des sirènes. Les mêmes policiers qui l'avaient battu plus tôt l'attrapent et le frappent à nouveau. S’ensuit une coupe franche sur le visage en gros plan de l'homme, Jim Collier, qui s'adresse au spectateur en ces termes, face caméra: «J'ai toujours voulu baiser une femme blanche» ${ }^{24}$. Tandis qu'il poursuit l'exposition de son fantasme et de sa première expérience sexuelle interraciale, le monologue est entrecoupé par l'image d'une femme blanche - d'abord une jambe et un visage en gros plan, puis étendue torse nu sur un lit, et enfin totalement nue sur le même lit. Il continue son histoire et les images de la femme deviennent de plus en plus suggestives; à un certain moment, elle commence à s'agiter sur le lit puis apparaît en gros plan, probablement du point de vue de l'homme, comme si elle faisait l'amour. Le son devient momentanément in lorsqu’elle gémit en atteignant un orgasme - s'ajoute alors en surimpression sur son visage une série de plans de plus en plus serrés sur Collier. Le montage raccorde ensuite la scène à une photographie de son visage en surimpression et sur ce qui semble être des images d'archive de manifestants pour les droits civiques, mais qui se trouve être en réalité des scènes de Come Back, Africa de Rogosin (fig.5).

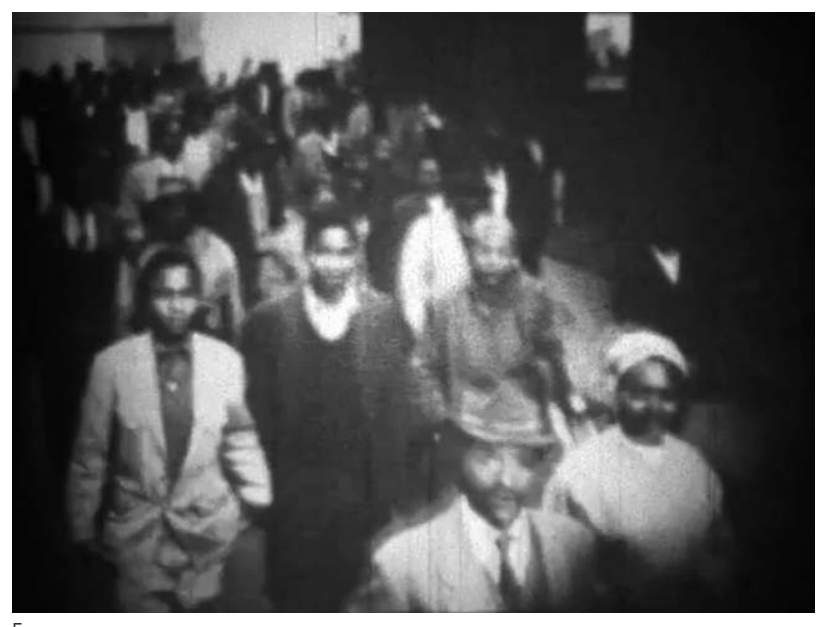

Image de Come Back, Africa, reprise dans Black Fantasy (1973)
24 Cette phrase liminaire de Black Fantasy ressemble à celle de The Cool World de Clarke, qui s'ouvre en très gros plan sur un harangueur de rue afroaméricain à Harlem clamant: "Voulezvous connaître la vérité sur l'homme blanc? L'homme blanc est le diable!». 
25 Le code Hays, qui interdit formellement le mélange des races, n'est totalement remplacé qu'en i968 par le système des âges. Ce n'est qu'après cette période que des contenus auparavant prohibés apparaissent dans le cinéma américain. Concernant les relations sexuelles interraciales, Hollywood esquive le sujet jusqu'à l'émergence de la blaxploitation, bien qu'elles soient davantage employées pour titiller le spectateur que comme un élément clé de l'intrigue.

26 Catalogue d'Impact Films, op. cit. [notre trad.].
Cette séquence d'ouverture donne le ton esthétique et politique de Black Fantasy, qui (malgré son contenu grivois pour l'époque ${ }^{25}$ ) montre Collier racontant son expérience personnelle, sociale et politique ainsi que le prix à payer lorsqu'on est un Noir marié à une femme blanche avec des enfants métisses dans une société raciste, un homme dont «toutes les pensées et sensations sont filtrées à travers une conscience raciale imposée à lui par la société et les circonstances ${ }^{26}$. Une grande partie du film alterne des entretiens de personnages face caméra racontant leurs histoires - Collier, son épouse et d'autres femmes - avec des images souvent en surimpression qui illustrent ou contredisent leurs récits. Les séquences additionnelles comprennent des inserts de manifestants, des scènes de rue à New York, des moments où Collier marche ou conduit une voiture à travers la ville ou la forêt ou encore des séquences de montage montrant une série de visages. Dans une autre séquence, Collier apparaît en gros plan et explique comment il s'est disputé avec une femme, qui pourrait avoir été son épouse, au sujet des raisons qui l'ont poussée à être en ménage avec lui. Alors qu’il se souvient de l'avoir accusée de «juste vouloir sa bite» et «sortir de la rigide société blanche», sa voix est perçue sur des images en surimpression de leurs deux visages et de plans sur des monuments célèbres à New York (fig. 6). La séquence se poursuit sur des images de Collier courant dans les bois, entrecoupées par des Blancs en

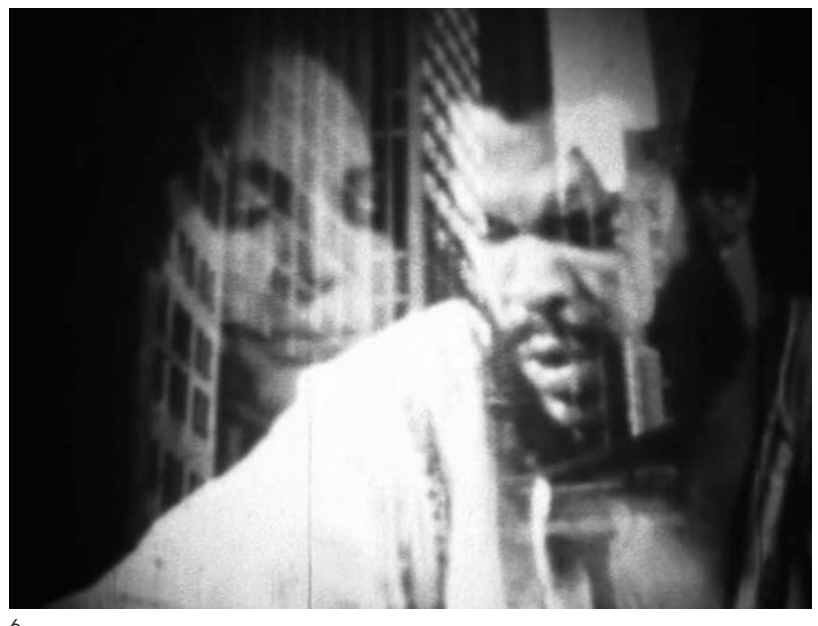


gros plan et accompagnées par des sons de tambours. Il se fait attraper et traîner par ce qui est devenu un groupe de Blancs prêts au lynchage. Les derniers plans alternent entre des images du groupe, le noud coulant d'une corde et un plan rapproché sur les pieds suspendus de Collier.

Alors que le sujet principal du film est celui des relations sexuelles entre Noirs et Blancs, cette thématique sert de tremplin à une exploration plus large des relations interraciales aux Etats-Unis et de ses effets sociaux et psychologiques sur ceux qui en sont particulièrement affectés. Comme le suggèrent les récits de Collier et des autres, l'histoire et l'expérience du racisme sont au cour de la plupart des relations interraciales, autorisant des fantasmes tout en affectant les individus et la société. Les liens que le film tisse entre désir et contrôle social atteignent leur apogée à deux reprises. Tout d’abord, comme le suggère la séquence abordée précédemment, lorsqu'une limite est tracée entre l'esclavage, la peur (des Blancs) du métissage des races et la violence raciale - en reliant les rapports sexuels entre un homme noir et une femme blanche avec la criminalité et la mort. Enfin, lorsque Collier évoque la crainte qu’il ressent pour ses enfants métisses, le film relie racisme et sexualité à des questionnements plus larges sur la durabilité de la famille noire.

Plus tard dans le film, après avoir débattu des relations entre femmes noires et blanches, Collier revient sur les difficultés économiques et sociales que de nombreux Noirs affrontent en tentant de maintenir leur famille intacte, et relie cette situation à sa propre expérience avec un père absent. Admettant tout d'abord haïr son père, il remarque son changement d'attitude, lorsqu'il devient adulte avec ses propres enfants:

«L'image normale d'un homme noir [...], du père noir [...], était celle d'un mec sans pouvoir. [...] Il ne pouvait avoir de travail qui lui permette de s'occuper de sa famille. [...] Au point que la meilleure chose qu'il lui restait à faire était de quitter sa famille. Au moins ils pourraient toucher l'aide sociale sans lui. [...] Quitter sa famille est une forme de suicide. J'entends par là, se séparer de la seule chose qu'on ait, [...] c'est une forme d'autopunition.»

Et lorsque Collier, cadré en plan moyen, fait part de ses pensées, des images du Jugement dernier de Jérôme Bosch (circa I482 ou après) apparaissent en surimpression. Une fois son histoire terminée, une coupe franche le montre en train de marcher dans les bois puis se coucher par terre. Ces plans sont entrecoupés par des images d'On the Bowery de 

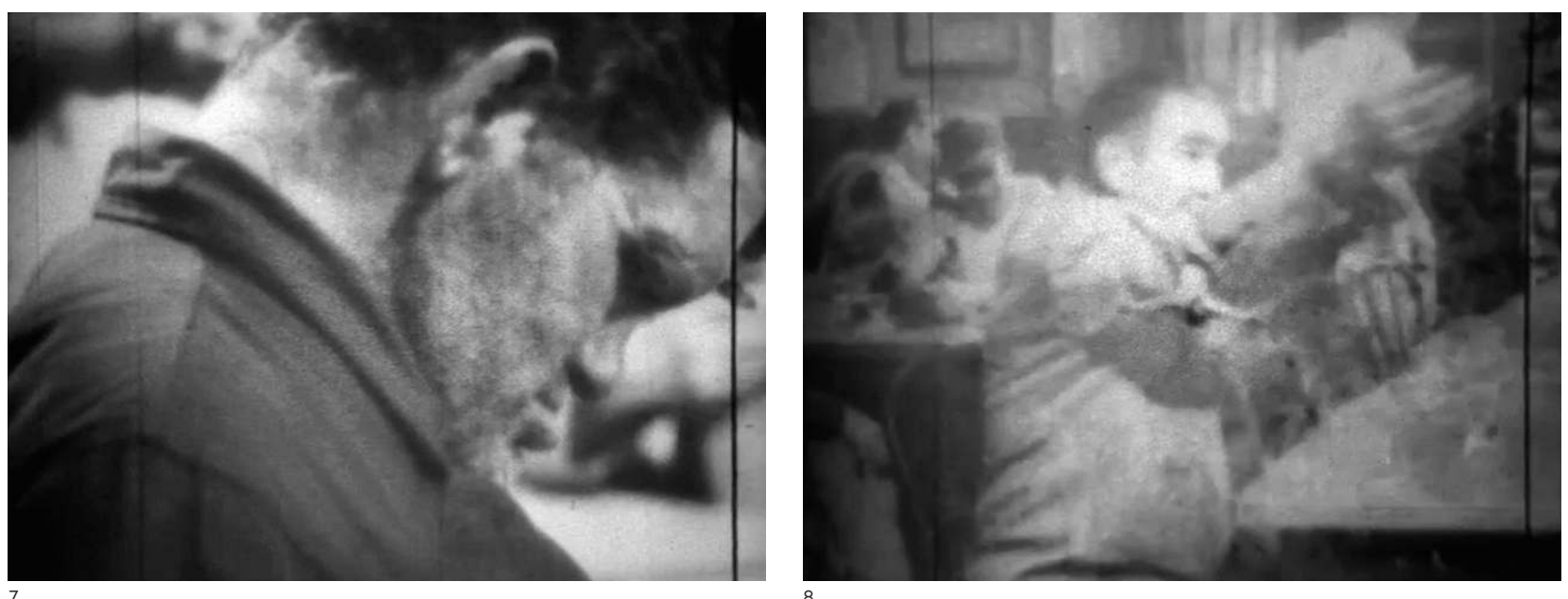

8

27 Daniel Eagan, America's Film Legacy: The Authoritative Guide to the Landmark Movies in the National Film Registry, New York, Continuum, 2010, p. 524 [notre trad.].
Rogosin et accompagnés de la bande-son du film précédent (fig.7 et 8). La majeure partie des plans tirés du premier film du réalisateur est extraite de ce que Rogosin appelle la «scène de folie», une longue séquence qui montre des hommes et des femmes dans un bar du Bowery qui deviennent de plus en plus saouls et turbulents en buvant du mauvais vin durant toute une soirée. Cette scène, selon Daniel Eagan, «était conçue, tournée et montée de telle manière qu'elle imite le désarroi et la perte de contrôle que l'ivrognerie entraîne ${ }^{27}$.

Alors pourquoi inclure des scènes d'un documentaire vieux de deux décennies et portant sur des Blancs alcooliques au sein d'un film expérimental sur la race et le sexe? Que ce soit pour redonner alors une visibilité à son premier long métrage oublié ou pour rallonger la durée de Black Fantasy, aucune de ces deux interprétations ne rend justice à ce moment marquant de forte réflexivité. On the Bowery tend à apporter à ses sujets contenance et humanité, leur offrant une voix malgré les circonstances. Une telle approche ne manque pas de susciter l'empathie des spectateurs. De la même manière, Rogosin, lorsqu'il collabore avec Collier, désire prolonger le sujet du métissage au-delà de la notion d'exploitation, offrant un aperçu historique personnel et complexe des liens entre persistance psychologique et sociale de l'esclavage et relations interraciales 
aux Etats-Unis. Plutôt que de constituer un traité sociologique sur la famille noire et les pères absents, un genre qui avait dominé durant la première moitié du $\mathrm{xx}^{\mathrm{e}}$ siècle ${ }^{28}$, le film relie le comportement à des facteurs historiques éloignés et communique, pour paraphraser Eagan dans un contexte différent, un sentiment de désarroi et de perte de contrôle plutôt que de débauche. Rogosin, pour sa part, relève des points communs entre désolation et désespoir, entre l'histoire de Collier et les hommes du Bowery.

Bien que Rogosin ne soit pas considéré comme un réalisateur newyorkais au même titre que Shirley Clarke l'est, il n'y a aucun doute sur l'influence qu'il a subie de par son implication avec des hommes et des femmes connectés au Groupe du Nouveau cinéma américain. Il n’a sûrement pas été aussi productif que certains, et il a peut-être choisi une autre direction relativement tôt dans sa carrière, mais ses interactions avec Jonas Mekas et avec d'autres, à quoi s'ajoute sa gestion du Bleecker Street Cinema, lui ont fourni les contacts et les connaissances qui ont influencé sa réalisation, que ce soit pour On the Bowery (très inspiré par Flaherty) ou pour le plus essayiste Black Fantasy. En résulte que, malgré le fait que nombre de ses films soient tournés à l'étranger, Rogosin est véritablement un réalisateur new-yorkais, possédant la capacité de relier le local au global.
28 Sur ce sujet, voir P. J. Massood, Making a Promised Land: Twentieth-Century African American Photography and Film, op. cit., pp. 88-I25. 